\title{
MECHANICAL DESIGN OF THE SNS COUPLED CAVITY ACCELERATOR*
}

\author{
N. Bultman, M. Collier, D. Richards, LANL, Los Alamos, NM 87545, USA
}

\begin{abstract}
The mechanical design and manufacturing details of the Spallation Neutron Source Coupled Cavity Linac are presented. The mechanical design and manufacturing approach for the RF cavity components is and results of initial development work for manufacturing is presented. The RF cavity components include both the main accelerating cavity structure (RF Segments) and the bridge couplers. The design utilizes a relatively standard approach for the RF accelerating segments but novel packaging techniques for the bridge coupler allow a minimum length coupler to be used. The resulting structure allows for a short magnetic focusing period and straightforward tuning of the multi-segment module.
\end{abstract}

\section{INTRODUCTION}

The Spallation Neutron Source has the goal of designing, fabricating, installing and commissioning a complete high-energy $\mathrm{H}^{-}$linac system at Oak Ridge National Laboratory to be used for the purpose of generating neutrons for materials research. The highenergy Linac spans an energy range from $2.5 \mathrm{MeV}$ to 1 $\mathrm{GeV}$ and is composed of a room temperature section consisting of a Drift Tube Linac (DTL), and a Coupled Cavity Linac (CCL), and a Superconducting section. The CCL section is a RF Linac, operating at $805 \mathrm{MHz}$ that accelerates the beam from 87 to $186 \mathrm{MeV}$ and has a physical installed length of slightly over 55 meters.

\section{SYSTEM CONFIGURATION}

The accelerator is modularized around a $5 \mathrm{MW} F$ power amplifier system. This requirement produces a CCL configuration of four modules, each containing 12 accelerator segments and 11 bridge couplers. A crosssection view of a subset of components is shown in Figure 1. The Accelerating structure is of the "Side-Coupled Cavity" variety. The segments are joined by bridgecouplers to form a continuous RF resonator. Electromagnet quadrupoles and beam diagnostic devices also occupy the spaces between the segments. The focusing period selected is 13-B $\lambda$ in a FDFD configuration; this gives $6.5-\mathrm{B} \lambda$ between magnet centers. Each segment occupies $4-\mathrm{B} \lambda$ and the remaining $2.5-\mathrm{B} \lambda$ is available for magnets and diagnostics. The bridge couplers are also located in the segment gaps on an offset from the beam centerline enough to provide space for the magnets and diagnostics to fit around the beamtube.

\subsection{RF Cavity Configuration}

Each segment has 8 accelerating cells and 7 coupling cells. The ends of each of the 10 internal segments to every module have a half-coupling cell that provides interface and power coupling with the bridge coupler. The bridge coupler then consists of the remaining halfcoupling cell and a longer center cell. In total each module consists of 213 cells. The RF power is fed into the module at two locations, into the center cell of segments 3 and 9 . This spacing provides roughly even power flow in both directions from each feed. The fact that the center cell in each coupler is excited provides the possibility for this convenient configuration. Within each segment, all of the cells are constrained to be the same length on the manufacturing drawings. This represents a reasonable compromise between exact phase match and a

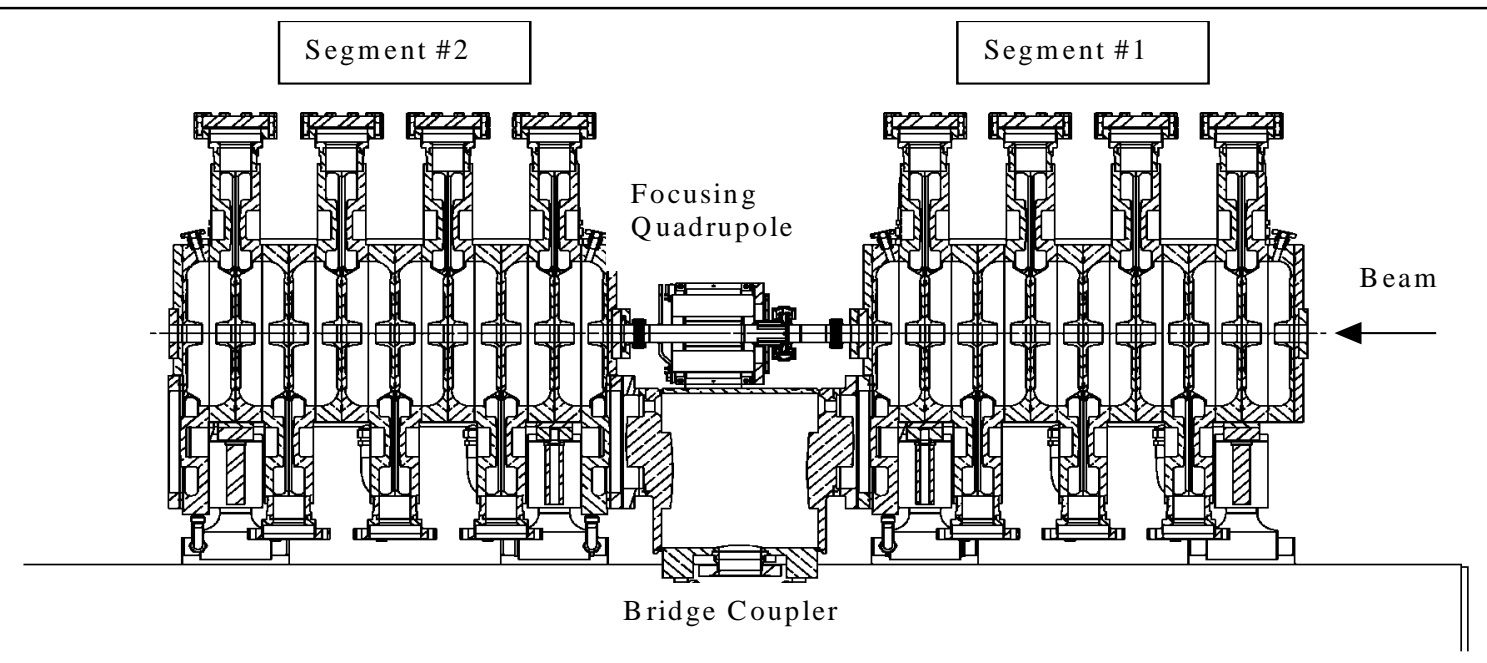

Figure 1. Cross-section view of the first two segments and first bridge coupler of the CCL 
configuration that can be more easily manufactured.

\begin{tabular}{|c|c|c|c|c|c|}
\hline \multicolumn{7}{|c|}{ Table 1. Module Design Parameters } \\
\hline Module \# & $\begin{array}{c}\text { Length } \\
(\mathbf{m})\end{array}$ & $\begin{array}{c}\text { Energy In/Out } \\
(\mathbf{M e V})\end{array}$ & $\begin{array}{c}\text { Energy } \\
\text { Gain (MeV) }\end{array}$ & $\begin{array}{c}\text { Avg. Gradient } \\
(\mathbf{M V} / \mathbf{m})\end{array}$ & $\begin{array}{c}\text { Module RF } \\
\text { Power (MW) }\end{array}$ \\
\hline 1 & 12.08 & $86.83 / 107.16$ & 20.33 & 1.68 & 2.82 \\
\hline 2 & 13.35 & $107.16 / 131.14$ & 23.98 & 1.80 & 3.30 \\
\hline 3 & 14.45 & $131.14 / 157.21$ & 26.07 & 1.80 & 3.42 \\
\hline 4 & 15.48 & $157.21 / 185.63$ & 28.42 & 1.84 & 3.56 \\
\hline Total & $\mathbf{5 5 . 3 6}$ & - & $\mathbf{9 8 . 8 0}$ & & $\mathbf{1 3 . 1 0}$ \\
\hline
\end{tabular}

Table 1 shows the general physical parameters of the CCL modules and the basic RF power requirements for cavity and beam power without any factors required for RF control margins applied. As previously mentioned, each module is supplied with a 5-MW Klystron so the typical $20 \%$ RF control margin to allow for waveguide and window losses as well as general control system requirements is satisfied.

\subsection{Support Systems Configuration}

The vacuum, water and mechanical supports and alignment equipment for the segments and quadrupole magnets are all attached to the welded steel support frame that consists of rectangular steel tubing and cross-braces. The vacuum manifold runs the continuous length of the structure with ports attached to the side coupling cells and the bridge couplers through formed steel bellows. The manifold is attached to the steel support structure and the vacuum pumps are attached directly under the manifold, also through formed steel bellows. The steel bellows allow for the differential thermal expansion between the manifold/support structure and the copper accelerator structure. The copper accelerator forms a continuous physical structure and thus is isolated from the steel support stand through linear bearings to allow for thermal expansion without stressing the accelerator cavities. The copper segments are attached to the linear bearings through steel cross members that bear on the underside of the copper cavities near the end cells of each segment. The linear bearings mount directly to the top of the steel weldment, and the cross-members allow for physical alignment of the segments to the beamline. The interface between the steel cross members and the linear bearings also incorporates a flexure on the tunnel wall side of the linac which allows for a small amount of transverse expansion difference between the steel structure and the copper cavities. The linear bearing centerline on the aisle side of the linac controls the transverse position of the assembly. This is referred to as the "master side" for alignment and the wall side as the "slave side".

\section{MANUFACTURING OVERVIEW}

The CCL segments are designed to be manufactured from simple hot rolled plate without the need for more expensive forgings. In addition the concept of a monolithic halfcell is utilized to allow incorporation of cooling water passages into the septum surfaces between cells while simultaneously eliminating the need for any additional braze joints between the side coupling cell and the main accelerating cell. This design approach allows for very simple and straightforward machining processes at the various steps with well-defined and accessible fixturing and hold-down points readily available to the machinist at each step.

\subsection{Manufacturing Steps Utilized}

a) Manufacturing begins with the production of the cell blanks from hot-rolled copper plate stock. The material is high purity Oxygen Free Electronic Grade (OFE) Copper that is certified to comply with the ASTM F-68 specification of Class II or Class I (Class II or better). This specification controls primarily the chemical composition, especially oxygen content limits (less than 5 PPM oxygen) and the wrought properties of the material.

b) The cell blanks are then finish machined on the septum side, Figure 2, to produce the coupling cell geometry and the septum cooling channels. The individual plates are called "half-cells".

c) In the next step, the half-cells are furnace brazed together in pairs along the septum surfaces in a vacuum furnace to produce rough "half-cell" assemblies. Alloy 50\% $\mathrm{Au}-50 \% \mathrm{Cu}$ is utilized with a braze temperature of $1850 \mathrm{~F}$. To this point all halfcell assemblies for each module are identical and can be parallel-processed at the maximum rate available

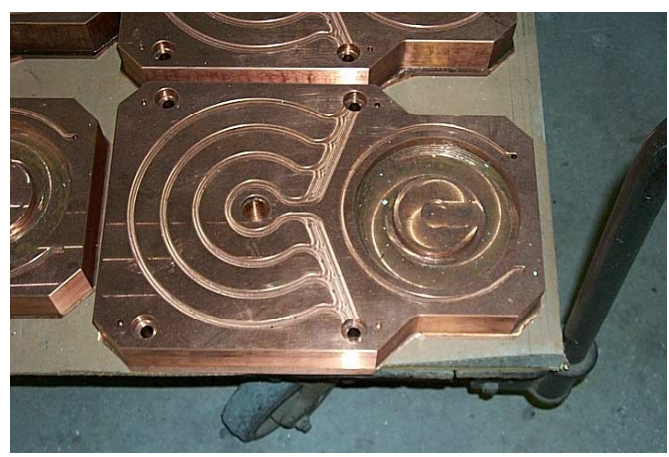

Figure 2. Completed half-cell septum

from the suppliers; parts are identified and distinguished only according to material lot and rolling heat number. This allows for later immediate identification and, if ever required, quarantine of all parts of a material batch if any subsequent process 
problems point to material defects. The rough halfcell assemblies vary between modules only in thickness; the plate material thickness is graded by module to reduce the volume of machining required in later steps of the process.

d) After helium leak testing, the accelerating cell sides of the plates are machined, Figure 3, to produce "finished half-cell-assemblies". The accelerating cells are machined directly to finished profile and the cavity contour includes a "tuning ring" that is used later during segment tuning. The first step of the cavity machining is standard lathe turning utilizing a simple counterweight for part balance on the faceplate. The second step involves milling the edge of the coupling slot connecting the accelerating cell and the previously produced side-coupling cell. This milling eliminates the thin sharp edge between the cavities and establishes a precisely specified opening geometry; this shape controls the RF coupling between the cells and thus field uniformity and stability through the module.

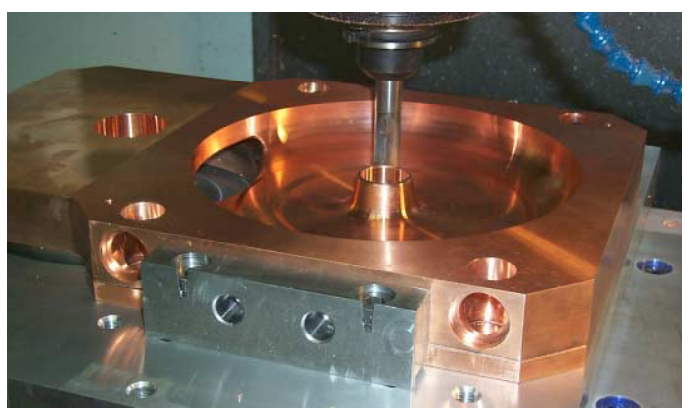

Figure 3. Machining tuning ring on finished end cell.

e) Following cavity finish machining the parts are again vacuum leak tested and cleaned thoroughly with a water-detergent solution and dried thoroughly with clean compressed air. The cells are now location specific by segment and are not interchangeable between segments. The cells are then stacked together in a special handling fixture, Figure 4, and the individual cell frequencies are measured and the accelerating mode frequency is determined. The required accelerating mode frequency must finally be established at $805 \mathrm{MHz}$ by removing material (machining on lathe or milling machine) from the tuning ring mentioned earlier. The parts are then thoroughly cleaned and stacked for brazing.

f) The stacked assembly is furnace brazed again using a copper-silver eutectic alloy at $1450 \mathrm{~F}$ in a vacuum furnace, Figure 5. This braze produces a finished but not tuned segment assembly complete with all water and vacuum fitting tube connections. The assembly is again vacuum leak tested and the final welds are made to vacuum connection flanges.

*Work supported by the Office of Basic Energy Science, Office of Science of the Department of Energy, and by Oak Ridge National Laboratory.

\section{CONCLUSIONS}

The selected design and manufacturing processes for the CCL accelerator segments has been reviewed and demonstrated through the steps of construction of various full scale copper manufacturing models. We believe that the design and manufacturing techniques are sound and represent an economical method of construction for the accelerator hardware.

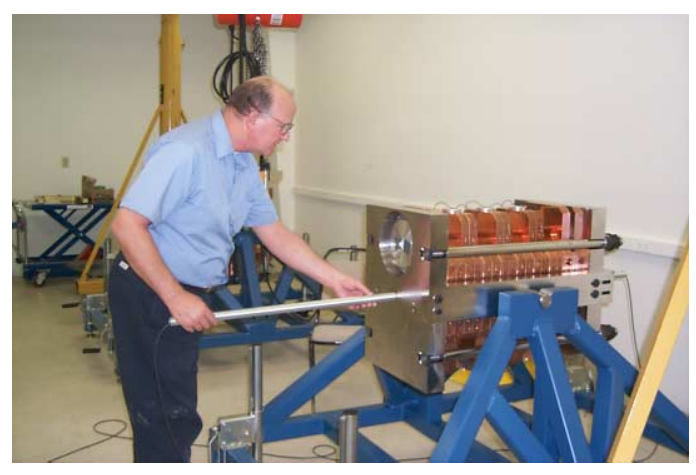

Figure 4. Frequency measurement

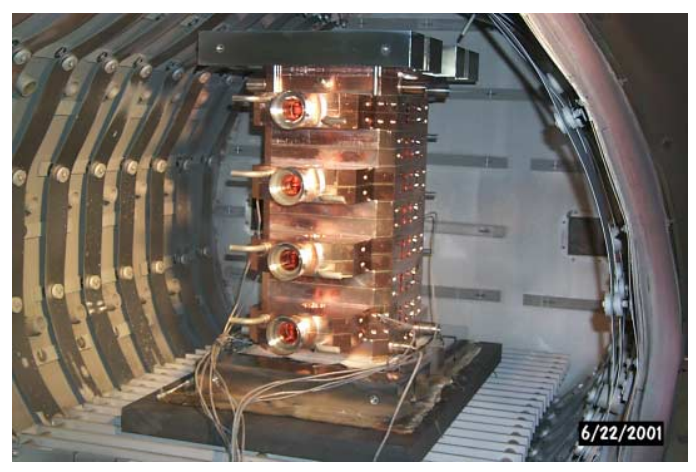

Figure 5. Stack brazing of segment assembly in the vacuum furnace. 